\title{
MINIMIZING SETUPS FOR CYCLE-FREE ORDERED SETS
}

\author{
D. DUFFUS, I. RIVAL AND P. WINKLER
}

\begin{abstract}
A machine performs a set of jobs one at a time subject to a set of precedence constraints. We consider the problem of scheduling the jobs to minimize the number of "setups".
\end{abstract}

Suppose a single machine is to perform a set of jobs, one at a time; a set of precedence constraints prohibits the start of certain jobs until some other jobs are already completed. Any job which is performed immediately after a job which is not constrained to precede it, however, requires a "setup"-entailing some fixed additional cost. The problem is schedule the jobs to minimize the number of setups.

It is common to render "a set of precedence constraints on a set of jobs" as "an antisymmetric and transitive binary relation on a set," that is, "a (partial) ordering on a set." In this analogy a "schedule satisfying the precedence constraints" becomes "a linear extension of the ordered set" (of all jobs). The problem of minimizing the number of setups can be entirely recast as a problem concerning linear extensions of an ordered set. The problem itself is attributed in [2] to Kuntzmann (cf. [6]). Progress on the problem can be found in several papers including $[3,4$, and 7] and recently W. R. Pulleyblank [7] has shown that this problem belongs to that class of problems whose complexity is described as $N P$-hard.

For elements $a, b$ of an ordered set $(P, \leqslant)$ - simply written as $P$-we say that $b$ covers $a$ if $a<b$ in $P$ and $a \leqslant c<b$ implies $a=c$. Let $L$ be a linear extension of $P$; that is, a total ordering of the underlying set of $P$ such that $a<b$ in $L$ whenever $a<b$ in $P$. A 'setup for $L$ ' is an ordered pair $(a, b)$ of elements of $P$ for which $b$ covers $a$ in $L$ but $a \Varangle b$ (and hence also $a \ngtr b$ ) in $P$. Let $s_{L}(P)$ count the number of such ordered pairs and let

$$
s(P)=\min \left\{s_{L}(P) \mid L \text { is a linear extension of } P\right\} .
$$

The problem is construct a linear extension $L$ of the ordered set $P$ for which $s_{L}(P)=s(P)$.

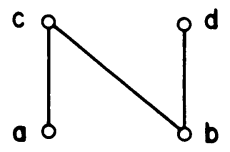

$\mathbf{P}$

$s(P)=1$

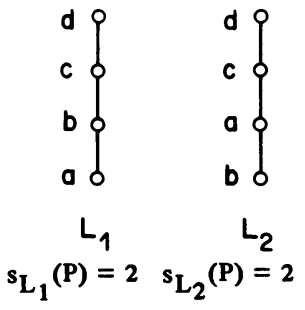

FIGURE 1
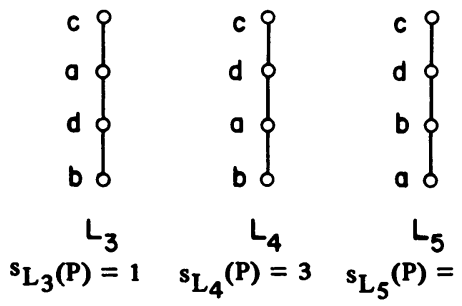

$s_{L_{5}}(P)=2$

Received by the editors June 12, 1981 and, in revised form, December 21, 1981.

1980 Mathematics Subject Classification. Primary 06A10. 
Any linear extension $L$ of $P$ can be obtained by partitioning $P$ into chains (linearly ordered subsets) $C_{1}, C_{2}, \ldots, C_{m}$ such that $x<y$ in $L$ if either $x<y$ in $P$, or $x \in C_{i}$ and $y \in C_{j}$, where $i<j$. In particular, $L$ is the linear sum of chains

$$
L=C_{1} \oplus C_{2} \oplus \cdots \oplus C_{m} \text {. }
$$

If the greatest element $\max \left(C_{i}\right)$ of $C_{i}$ is not below the least element $\min \left(C_{i+1}\right)$ of $C_{i+1}$ in $P$, then $\left(\max \left(C_{i}\right), \min \left(C_{i+1}\right)\right)$ is a setup for $L$. Evidently, $s_{L}(P) \leqslant m-1$ and if $\max \left(C_{i}\right) \neq \min \left(C_{i+1}\right)$ for each $i=1,2, \ldots, m-1$, then $s_{L}(P)=m-1$. According to Dilworth's theorem [5], the smallest number of chains into which $P$ can be partitioned is equal to the width $w(P)$ of $P$ - the size of a maximum-sized antichain. Therefore, $s(P) \geqslant w(P)-1$.

Of course, equality does not in general obtain. Indeed, a partition $C_{1}, C_{2}, \ldots, C_{w(P)}$ of $\boldsymbol{P}$ into chains can be arranged to form a linear extension of $\boldsymbol{P}$ only if there is a permutation $\rho$ of $\{1,2, \ldots, w(P)\}$ such that $\rho(i)<\rho(j)$ implies $x \ngtr y$ for any $x \in C_{\rho(i)}$ and $y \in C_{\rho(j)}$. No such permutation could exist if there were a subset (say, $\left.\left\{C_{1}, C_{2}, \ldots, C_{n}\right\}\right)$ of the partition, and elements $x_{i}, y_{i} \in C_{i}, i=1,2, \ldots, n$, satisfying

$$
y_{1}<x_{1}, x_{1}>y_{2}, y_{2}<x_{2}, x_{2}>y_{3}, \ldots, x_{n-1}>y_{n}, y_{n}<x_{n}, x_{n}>y_{1} \text {. }
$$

An ordered set $\left\{x_{1}, y_{1}, x_{2}, y_{2}, \ldots, x_{n}, y_{n}\right\}$ of size $2 n, n \geqslant 2$, with these comparabilities, and no others, is called an alternating $2 n$-cycle, or more briefly a $2 n$-cycle (see Figure 2).

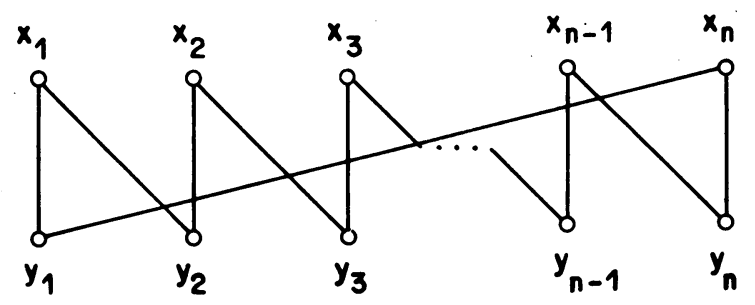

Figure 2

The ordered sets shown in Figure 3 are cycle-free, that is, contain no subset isomorphic to an alternating $2 n$-cycle.
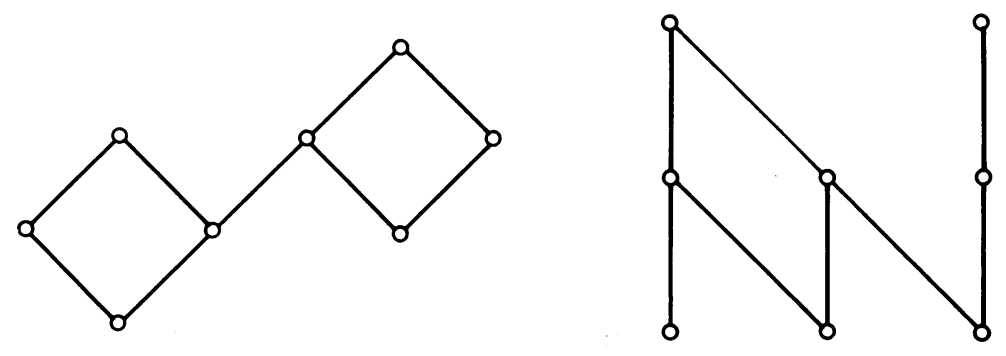

Figure 3 
The principal result of this paper is

THEOREM. Let $P$ be an ordered set without alternating cycles. Then $s(P)=w(P)-1$.

The case where $P$ has length two (that is, $P$ has no three-element chain) is particularly easy to verify. We proceed by induction on the size of $P$ : if $P$ contains an isolated element $a$ then $w(P-\{a\})=w(P)-1$ and clearly $s(P)=s(P-\{a\})$ +1 . Otherwise, as $P$ is cycle-free there is an element $b$ comparable with precisely one other element, say, $b<c$. Again if $w(P-\{b\})=w(P)-1$ then the induction hypothesis applies; otherwise, $w(P-\{b\})=w(P)$ and, indeed, $w(P-\{b, c\})=$ $w(P)-1$. Finally, $s(P)=s(P-\{b, c\})+1$, so in any case, $s(P)=w(P)-1$.

Before we turn to the proof of the theorem, note from the ordered sets illustrated in Figure 4 that the converse of the theorem cannot hold.

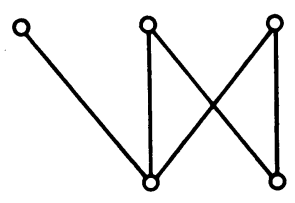

$P_{1}$

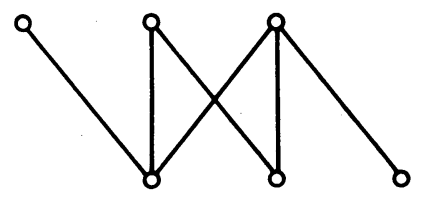

$P_{2}$

$$
s\left(P_{i}\right)=2=w\left(P_{i}\right)-1 \quad(i=1,2)
$$

\section{Figure 4}

Proof of the theorem. We proceed by induction on $m=w(P)$. Let $C_{1}, C_{2}, \ldots, C_{m}$ be a sequence of maximal chains of $P$ such that

$$
P=\bigcup_{i=1}^{m} C_{i} \text {. }
$$

(Such a sequence can always be obtained by extending each of the $m$ chains in a partition of $P$ by width-many chains.)

Let $x, y, z \in C_{i}$ with $x<y<z$ and suppose that for some $j,\{x, y, z\} \cap C_{j}=\{y\}$. Then some element $x^{\prime}$ in $C_{j}$ must be noncomparable to $x$, else the addition of $x$ would extend $C_{j}$; similarly there must be an element $z^{\prime}$ of $C_{j}$ noncomparable to $z$. But then $\left\{x, z, x^{\prime}, z^{\prime}\right\}$ is a 4-cycle, contradicting the hypothesis of the theorem. It follows that, for any $i$ and $j$ and any $y \in C_{i} \cap C_{j}$, either $\left\{x \in C_{i} \cup C_{j} \mid x \leqslant y\right\}$ is a chain or $\left\{z \in C_{i} \cup C_{j} \mid z \geqslant y\right\}$ is a chain.

For each $i$, let

$$
P_{i}=C_{i}-\bigcup_{j \neq i} C_{j}
$$

Then $P_{i} \neq \varnothing$ for each $i=1,2, \ldots, m$, for otherwise $m=w(P)<m$. We now introduce a binary relation " $\rightarrow$ " on $\left\{C_{i} \mid i=1,2, \ldots, m\right\}$ as follows: $C_{i} \rightarrow C_{j}$ if there are elements $x \in P_{i}$ and $y \in C_{j}-C_{i}$ such that $x>y$ in $P$. The definition is motivated by this observation:

$$
\text { if for some } i, C_{i} \nrightarrow C_{j} \text { for all } j \text { then } s(P)=w(P)-1 \text {. }
$$


To prove this let $x=\max \left(P_{i}\right), C=\left\{y \in C_{i} \mid y \leqslant x\right\}$, and let $P^{\prime}=P-C$. Then $w\left(P^{\prime}\right)=w(P)-1$ and by the induction hypothesis there is a linear extension $L^{\prime}$ of $P^{\prime}$ consisting of a linear sum of $m-1$ chains of $P^{\prime}$. We claim $L=C \oplus L^{\prime}$ is a linear extension of $P$; if not, there are elements $y \in C$ and $z \in P^{\prime} \cap C_{j}$, for some $j \neq i$, with $y>z$. Hence $z<x$ and since $C_{i} \nrightarrow C_{j}$, it must be that $z \in C_{i}$; then $z \in C$, an impossibility.

We may therefore suppose that for each $i$ there is some $j$ such that $C_{i} \rightarrow C_{j}$. After suitable relabelling, there is a sequence $1,2, \ldots, n$ of smallest length such that $C_{1} \rightarrow C_{2} \rightarrow \cdots \rightarrow C_{n} \rightarrow C_{1}$.

Choose $x_{i} \in P_{i}$ and $y_{i} \in C_{i}-C_{i-1}$ with $x_{i}>y_{i+1}$, for each $i=1,2, \ldots, n(\bmod n)$. Observe that $x_{i}>y_{i}$ for each $i, 1 \leqslant i \leqslant n$. We conclude the proof by verifying that $\left\{x_{1}, y_{1}, x_{2}, y_{2}, \ldots, x_{n}, y_{n}\right\}$ must now contain an alternating cycle. Let us suppose that it is not itself a $2 n$-cycle.

Case (i). Let $x_{i}>x_{j}$. Since $x_{i} \notin C_{j}$ there is some $x>x_{j}$ in $C_{j}$ which is noncomparable with $x_{i}$. Further, since $y_{j+1} \notin C_{j}$ there is some $y<x_{j}$ in $C_{j}$ which is noncomparable with $y_{j+1}$; then $\left\{x_{i}, x, y_{j+1}, y\right\}$ is a 4-cycle.

Case (ii). Let $y_{i}=y_{j}, i \neq j$. Then $C_{i-1} \rightarrow C_{j}$, contradicting the minimality of $n$.

Case (iii). Let $y_{i}<y_{j}$. If $y_{i} \notin C_{j}$ then there is $y<y_{j}$ in $C_{j}$ noncomparable with $y_{i}$, so $\left\{x_{j-1}, x_{j}, y_{i}, y\right\}$ is a 4-cycle. If $y_{i} \in C_{j}$ then $C_{i-1} \rightarrow C_{j}$, again contradicting the minimality of $n$.

It follows that $y_{i}$ is noncomparable with $y_{j}$ for each $i \neq j$.

Case (iv). Let $x_{i}>y_{j}$, where $j \neq i$ and $j \neq i+1$. Since $y_{j}$ is noncomparable with $y_{i}$, $y_{j} \notin C_{i}$ so $C_{i} \rightarrow C_{j}$ which is again impossible.

Case (v). Let $x_{i}<y_{j}$. Then $y_{i}<y_{j}$ which was already ruled out.

This completes the proof.

An algorithm. Implicit in the proof of the theorem is an algorithm to construct a linear extension $L$ of a cycle-free ordered set $P$ which is optimal in the sense that $s_{L}(P)=s(P)=w(P)-1$. The following procedure, though inductive, is based on a single covering $C_{1}, C_{2}, \ldots, C_{w(P)}$ of $P$ by maximal chains.
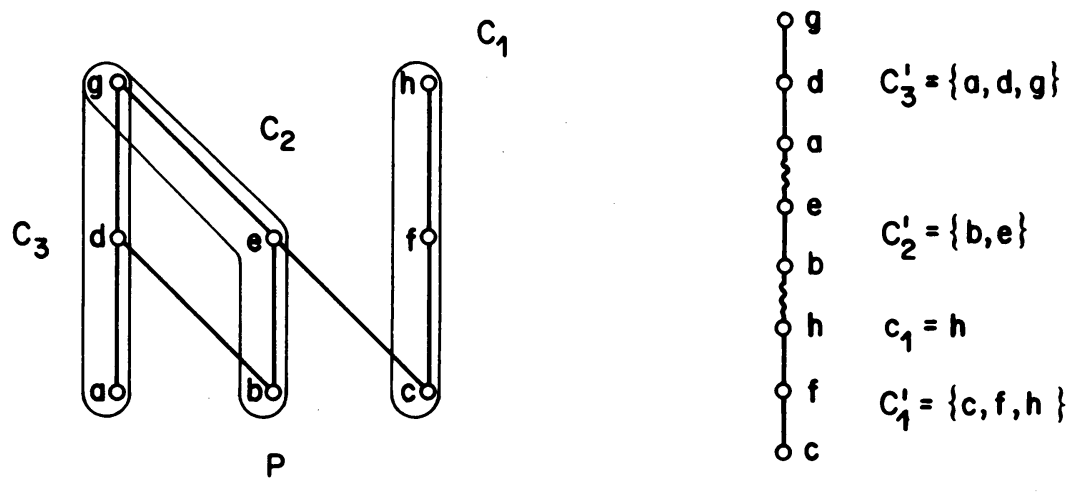

FIGURE 5 
According to the proof of the theorem, in any such covering there is a chain (say, $C_{1}$ ) such that for any $i=2,3, \ldots, w(P), C_{1} \nrightarrow C_{i}$. Let $c_{1}=\max \left(P_{1}\right), C_{1}^{\prime}=\left\{x \in C_{1} \mid\right.$ $\left.x \leqslant c_{1}\right\}$, and $Q=P-C_{1}^{\prime}$. Then $Q$ is covered by the chains $Q \cap C_{2}, \ldots, Q \cap C_{w(P)}$, and by inductive use of this algorithm $Q$ has a linear extension

$$
L^{\prime}=C_{2}^{\prime} \oplus C_{3}^{\prime} \oplus \cdots \oplus C_{w(P)}^{\prime}
$$

with $s_{L^{\prime}}(Q)=w(Q)-1$, where $C_{i}^{\prime} \subset C_{i}$ for each $i=2,3, \ldots, w(P)$. Then $L=C_{1}^{\prime} \oplus$ $L^{\prime}$ is a linear extension of $P$ for which $s_{L}(P)=w(Q)=w(P)-1$ as required.

The algorithm is illustrated in Figure 5 for a particular cycle-free ordered set of width three.

\section{REFERENCES}

1. G. Chaty, M. Chein, P. Martin and G. Petolla, Some results about the number of jumps in circuit digraphs, Proc. Fifth Southeastern Conf. Combinatorics, Graph Theory and Computing, Utilitas Math., Winnipeg, 1974, pp. 267-279.

2. M. Chein and P. Martin, Sur le nombre de sauts d'une forêt, C. R. Acad. Sci. Paris 275 (1972), 159-161.

3. M. Chein and H. Habib, The jump number of dags and posets: an introduction, Ann. Discrete Math. 9 (1980), 189-194.

4. O. Cogis and M. Habib, Nombre de sauts et graphes série-parallèles, RAIRO Inform. Théor. 13 (1979), 3-18.

5. R. P. Dilworth, A decomposition theorem for partially ordered sets, Ann. of Math. 51 (1950), 161-166.

6. J. Kuntzmann and A. Verdillon, Recherche d' un ordre total minimal compatible avec un ordre partial donné, Séminaire Institut de Mathématique de Grenoble, 1971.

7. W. R. Pulleyblank, On minimizing setups in precedence constrained scheduling, Discrete Appl. Math. (to appear).

Department of Mathematics, Emory University, Atlanta, Georgia 30322

Department of Mathematics, University of Calgary, Calgary, T2N 1N4, Alberta, Canada 